\title{
A Study on Light Embodiment Method through Technology Analysis of Light Art
}

\author{
Kim, Byung-kyu \\ Media Artist \\ camacart@daum.net
}

\begin{abstract}
Emitted light through lighting became an aesthetic object itself, and lighting became the driving force and desire of artwork creation to artists. Specially, severatantists tried to give shape to the pure substance radiating from lighting fixtures, not the light containing lighting fixtures, in empty space. They intended to control light that is difficult to control physically. James Turrell, Anthony McCall, and Troika are the ones who thade on experiment on such light, and they tried to shape light itself through their own method, The AT Field_Paralyzed Sense I made has an interactive installation format as an artwork that intended to materialized light not just as a visual object, but as a physicabobject that can be experienced through sense of touch as well through lesser characteristics. The AT Field_Paralyzed Sense materialized three-dimensional modeling of light with diffraction restrictive characteristic of laser, characteristic of getting reflected on mirror, and visualizing characteristic of emitted laser light on the rotating mirror surface in high speed on the surface of light. The modeling technology method of light of the AT FFeld_Paralyzed Sense using laser not only accomplished physical modeling of pure $\sqrt{1 g h t}$ which had been desire of artists for long time, but also suggests the possibility as a nem formative language in artistic aspect as well.
\end{abstract}

Keywords: Light Art, Media Art, Immersive Environment, Light Embodiment

\section{Introduction}

Experiments on light sarted to develop in earnest as an aesthetic element through a genre of art, called the light an The genre became possible when technology of human beings of controlling light became possible. It was because artists who used light as a material of artwork or as an aesthetic object perceived light as aesthetic elements within in a material called light.

There are tyo aspects of what art forms using light are seeking for. The first is formative aspect an intention to create aesthetic forms by perceiving light as a new visual material. It is because the new form itself connotes new aesthetic value. The second is content aspect for delivering ideas of artists by using form characteristics of light, and light was similar to a vehicle of delivering contents.

Artists who tried to expand new art form with light had no other choice but to use visual elements other than light overwhelmingly as the form of artwork. This was an intention to create work using elements as the form of lighting fixture, colors contained in light, and form of space with projected light, and the pure light itself was not used as a modeling element. Light was only molded indirectly through mediators, including light. However, several artists only tried to mold light through pure substance itself, the light, and the result of such effort was evident in several artworks.

This study researched cases of using light as a pure material, and intended to analyze the relationship between light as an aesthetic element and form that appeared in each work. This 
study will research how artists who only tried to use light as a pure material achieved the purpose to which level. The intention was to identify artistic desire of artists of giving body to an untouchable material, the light, as a touchable object through visual forms. Also, this study will research how the light modeling as a touchable physical object became realized through which technical method by analyzing modeling elements of light revealed through an interactive media art that I made myself, the AT Field_Paralyzed Sense. Therefore, the modeling possibility of using pure light appeared through technical method of AT Field_Paralyzed Sense will suggest new technical solutions to other artists who wish to create artworks with light.

\section{Light as an Artistic Material}

It was not easy to use light as an aesthetic element before equipments, as lighting, that can contain light artificially were developed. Historically, moulded visua/ objects using light indirectly started to appear in British Christian Church and Islamic architectures of 7 th century, and light was not used directly, but used visua pbenoment which take place when sunlight passes through materials. For example, stained glass is formed with geometric, decorative form, or pictorial design on colored glass or glass with colored surface, and this became the first art form using light in history of art. Stained glass is an art form using light, but it is an art form of different property ofrom light art in terons of how it can only become visualized when a medium of laws of nature, called sunlight, exists, and in terms of visualization through mediated light, nof the direct aesthetic object.

As László Moholy-Nagy stated "this century belongs to light" in 1927, and as light was used as an art form and art object through artworks as 'Light-Space Modular' that he created in 1922, forms of art created with light becamea new genre of visual art. However, light in artworks by Nagy was used as an additive material that highlights the changing form more, and treated light as one of the changing aesthetic materials. Therefore, art form of Nagy is treated in the kinetic art genre that conducts movement as an art object.

It was the 1960s when ligh was recognized as a material and topic in earnest and experiments on light as a new media took place. Dan Flavin and James Turrell were the first artists who used Hight actively)as an aesthetic material. This chapter will analyze aesthetic elements of light and review efforts of artists who actively used light to visualize light itself by excluding other external factors to suggest descriptive basis for the light art of a new form, called pure dimensionahity of light.

\subsection{Aesthetic Factor of Light}

Aesthetre elements of light as an aesthetic object can be classified into four large categories. The first is light intensity and the second is color radiated through light. The third is formative element seen through objects that contain or mediate light. The fourth is spatial element that appears as diffused light is projected on space. The last spatial element of light is an aesthetic element that cannot be found in works that made an object itself, the light, visualized. It is because cases of using scattered light that affects physical elements cannot use the fourth element as an aesthetic element. Therefore, the three elements, excluding the spatial element, always get involved in work formality as aesthetic elements. Outputs created through different physical control of these four elements arouse different aesthetic sensitivity from each other, and such sensitivity gets aroused through information accepted via human visual-perception process. 
Therefore, four aesthetic elements of light will be analyzed through precedence cases, and aesthetic characteristic of artworks that only visualized light as pure substance will be researched.

\subsection{Case Analysis on the Multi-Dimensional Modeling of Light}

2.2.1. James Turrell, Afrum: One of the earliest works of James Turrell, Afrum, appears like a cubical light. Light is projected in square shape towards the center of the corner of white interior space. As physical effect of making the image appear elongated when light gets drifted further is used, and the light in rectangular form makes cubical three-dimensional figure. Most of early works by James Turrell used a method of projecting light on the eorner of space. Not just the Afrum, but series created in this manner used a visual effect of making the two-dimensional light to appear three-dimensional through the space tructure of threedimensional wall. This represents his intention to substitute dighe as a physical object and to visualize it. Therefore, when it is viewed as a visual effect, Afrum appears as a lump of touchable light in geometric shapes made out of puresubstances. What formative elements in James Turrell's works reveal is the fact that visual elements in his works are solely composed with light. Nothing, other than light, is involved in his works as a component.

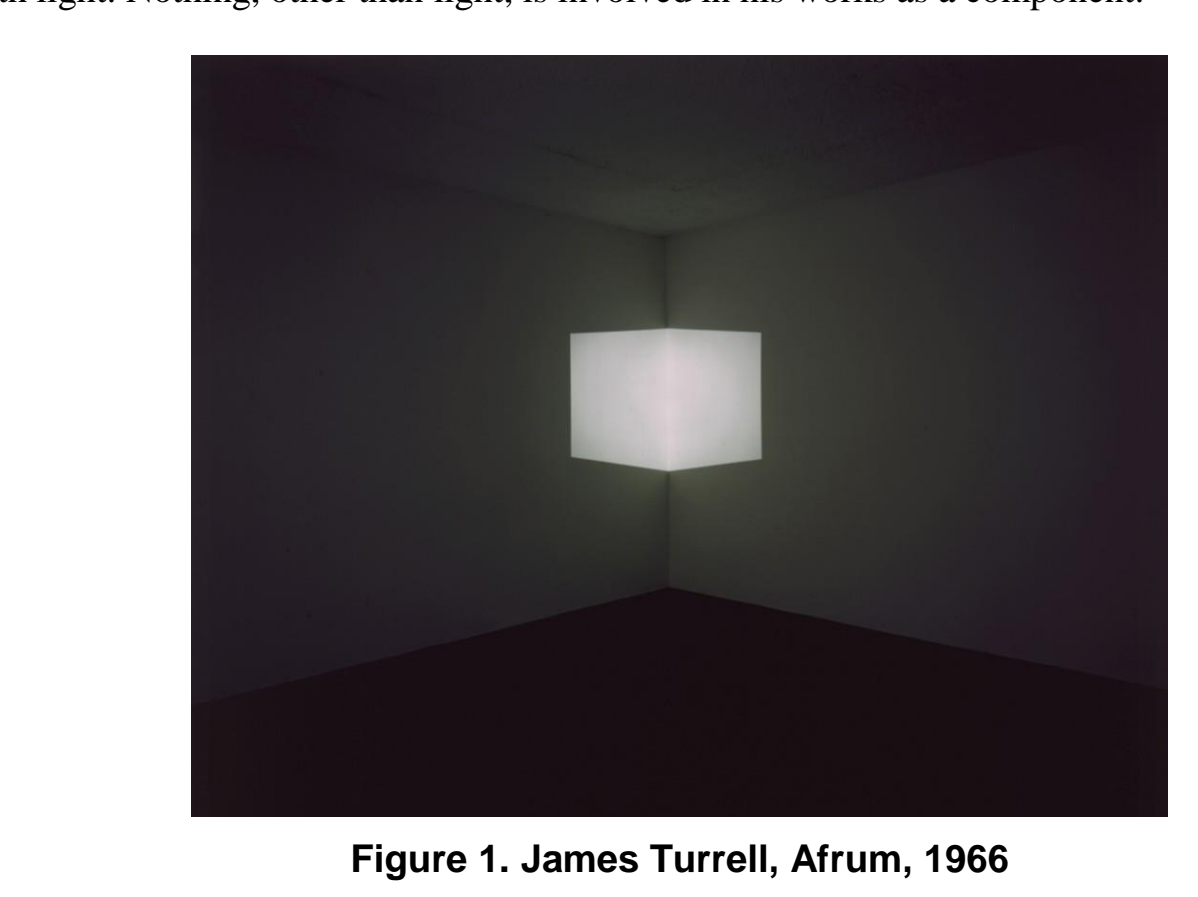

Previously defined the intensity of light as an aesthetic element appears as though Afrum was adjusted to the degree of not revealing the physical space, called corner, in space. It is because when physical space, the corner, gets revealed, it will not appear relatively as a lump of light in physical form. For color as a second aesthetic element, the white color of light was used. The third element is a medium of visualized light, and as explained above, the corner where walls are put together plays the role of medium that holds light together. The last is spacial element, and the third and fourth elements overlap as it appears through its relationship with corner, which is the third aesthetic element of light.

Through this analysis, it was identified that the intention and purpose of Afrum by James Turell was not fully accomplished in terms of how he only wanted to mould pure light. 
Because the visual derangement effect, called dimensionality of light, composed solely with pure light occurs due to a medium, called the corner.

2.2.2. Anthony McCall, Line Describing a Cone: The Line Describing a Cone, one of the Solid Light series by Anthony McCall is an installation that makes projected light from a projector to appear as a large lump of light in space. Literally, the projection of an image on a wall is a curved line, but it becomes three-dimensional as it leaves traces of a large form in dark space. Viewers will explore the space and experience the large lump of light.

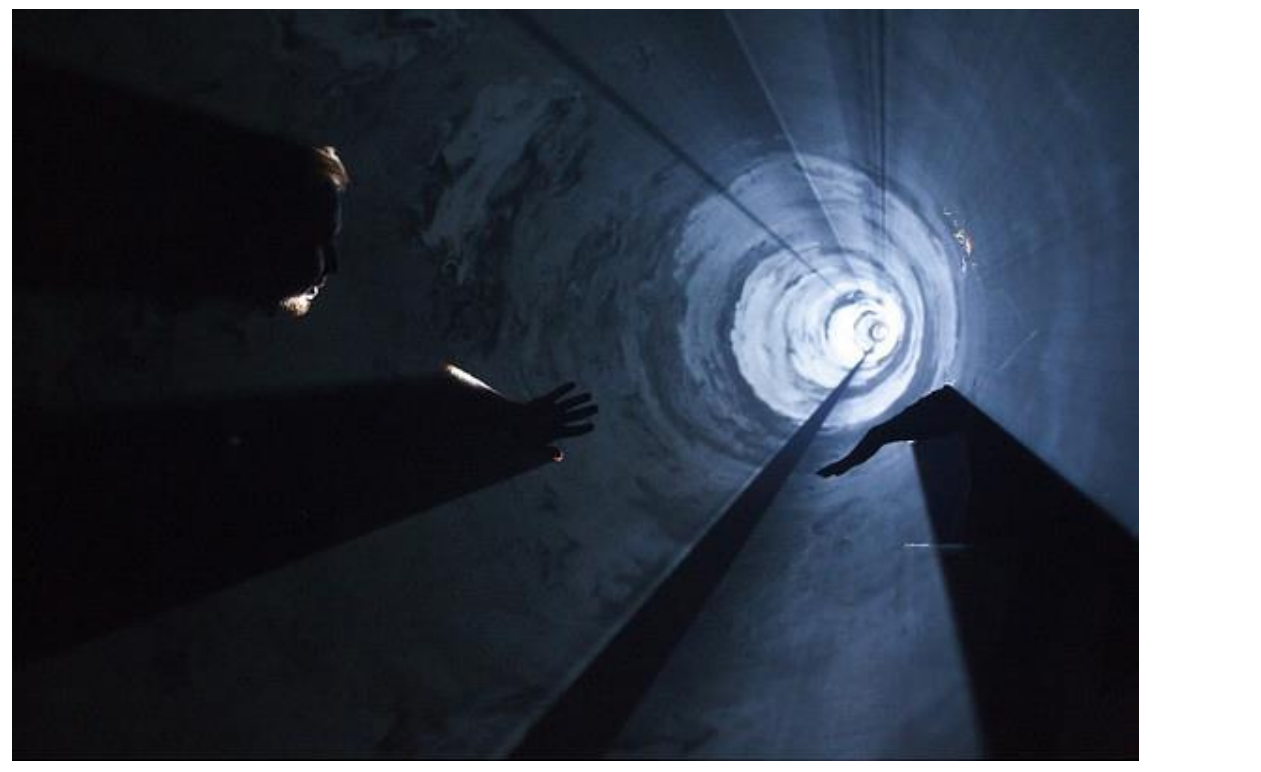

Figure 2. Anthony McCall, Line Describing a Cone, 1973

As an aesthetic lement, the intensity of light is very intense in a sense that light can be seen in dark space only. As most of works by Anthony McCall, the penetrated light from outside gets completely sbut off and only the pure light itself generated from his lighting installation becomes visualized in space. Therefore, as the structure of space cannot be accurately identified and only three-dimensional light displays its physical form in space, only the pure light becomes the molded art from. Of course, fog, the visualization medium of light, was sprayed in space to maximize the visualization of light.

There is one limitation in Anthony McCall's free moulding method that light gets determined by the distance from a light source. Therefore, the three-dimensional moulding of light cannot be accomplished when some distance is not ensured. Only the light installed in front of a light source forms the light depending on the cracked shape in a lighting fixture where the light smears out.

2.2.3. Troika, Arcades: Arcade is an installation work that arranged the arch-shaped light structures. Troika used lens to mould forms with light, and the lens is made out of general materials used for optical control of light that the motion direction of light changes according to its curved shape and makes physical control of light possible. A lens that penetrates light in straight direction is installed on a lighting fixture on the bottom of a column and the second lens is installed at some point where the light is flashed on. The second lens is designed for light to be bent in one direction when the flashed light passes through the lens. This is how 
the light gets bent. Therefore, when such lighting fixtures are arranged face to face, it will appear as an arch-shaped lighting structure.

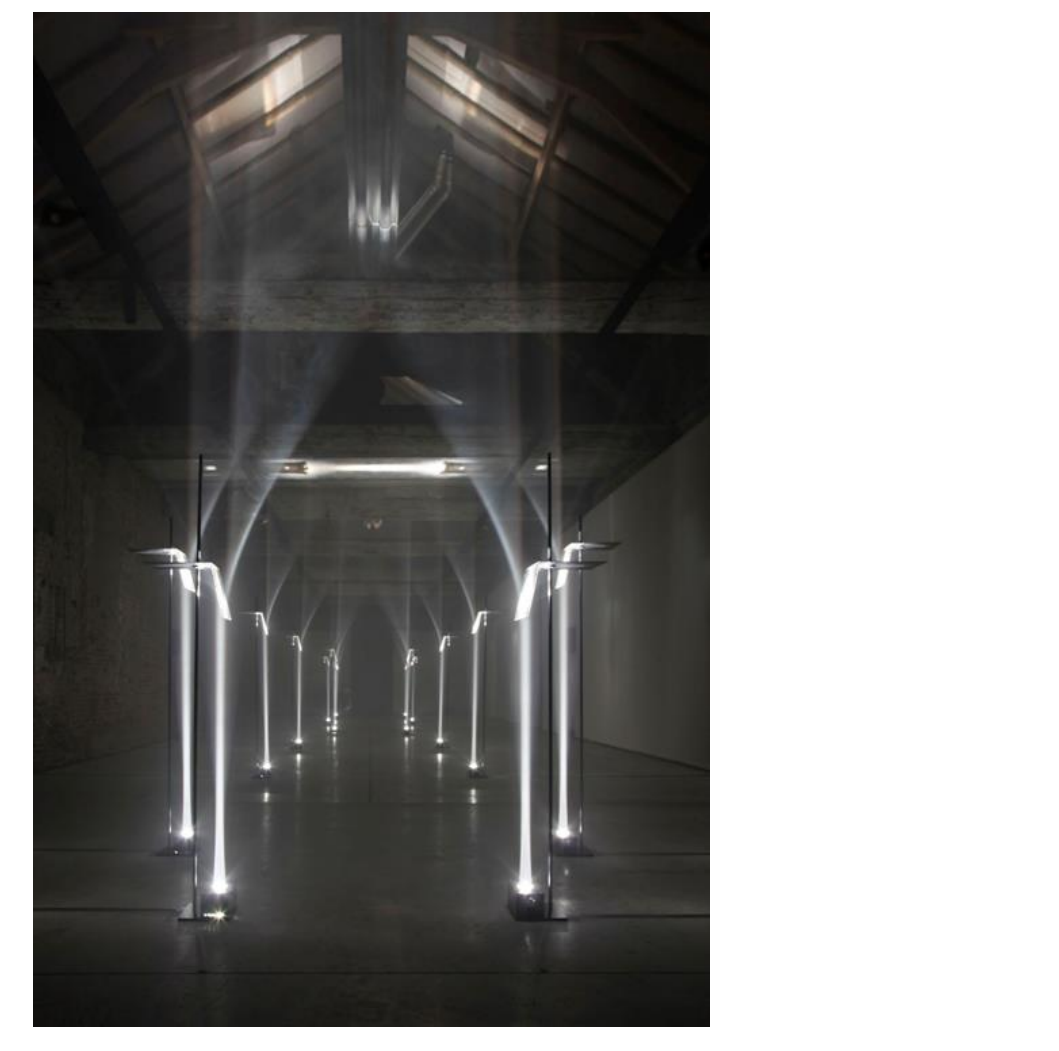

Figure 3. Troika, Arcades, 2012

The intensity of light in arcade blocked light from outside, but the inside where lighting is installed is enough to be recognized visually. The phenomenon occurs due to the characteristic of installed dighting fixture, and it is because the light has diffracting phenomenon in general. In morphological aspect, there is a visual phenomenon of light being kept in lens because lens is used in Troika to bend the light, but the light was successfully controlled physically and the significance is in how a pure substance as light was used for moulding similat to other works mentioned above.

The mosinteresting visual characteristic of Arcade is how light was bent physically by the lens structure. Torika also used fog, a medium of light in air, to maximize the visualization of light

2.2.4. Byungkyu Kim, AT Field_Paralyzed Sense: AT Field_Paralyzed Sense is an interactive installation. When viewer is seated on a chair placed within the cubical frame, film of light that surrounds viewer gets created and the viewer gets to experience the visual effect of getting trapped in space of light. Through this process, the viewer experiences immersive environment created with light. AT Field_Paralyzed Sense used laser to form the film of light.

Light in general has the diffracting characteristic. We can light up the space through the diffraction phenomenon of light. However, laser has a characteristic of limiting the diffraction. This is the reason why the wavelengths of light do not diffuse and show a distinct visual feature of shooting out clearly in straight line. AT Field_Paralyzed Sense used the effect of showing light as surface, instead of line, through characteristics of laser that limits 
diffusion, characteristic of getting reflected on mirror, and through the bonding structure with mirror revolving at high speed.

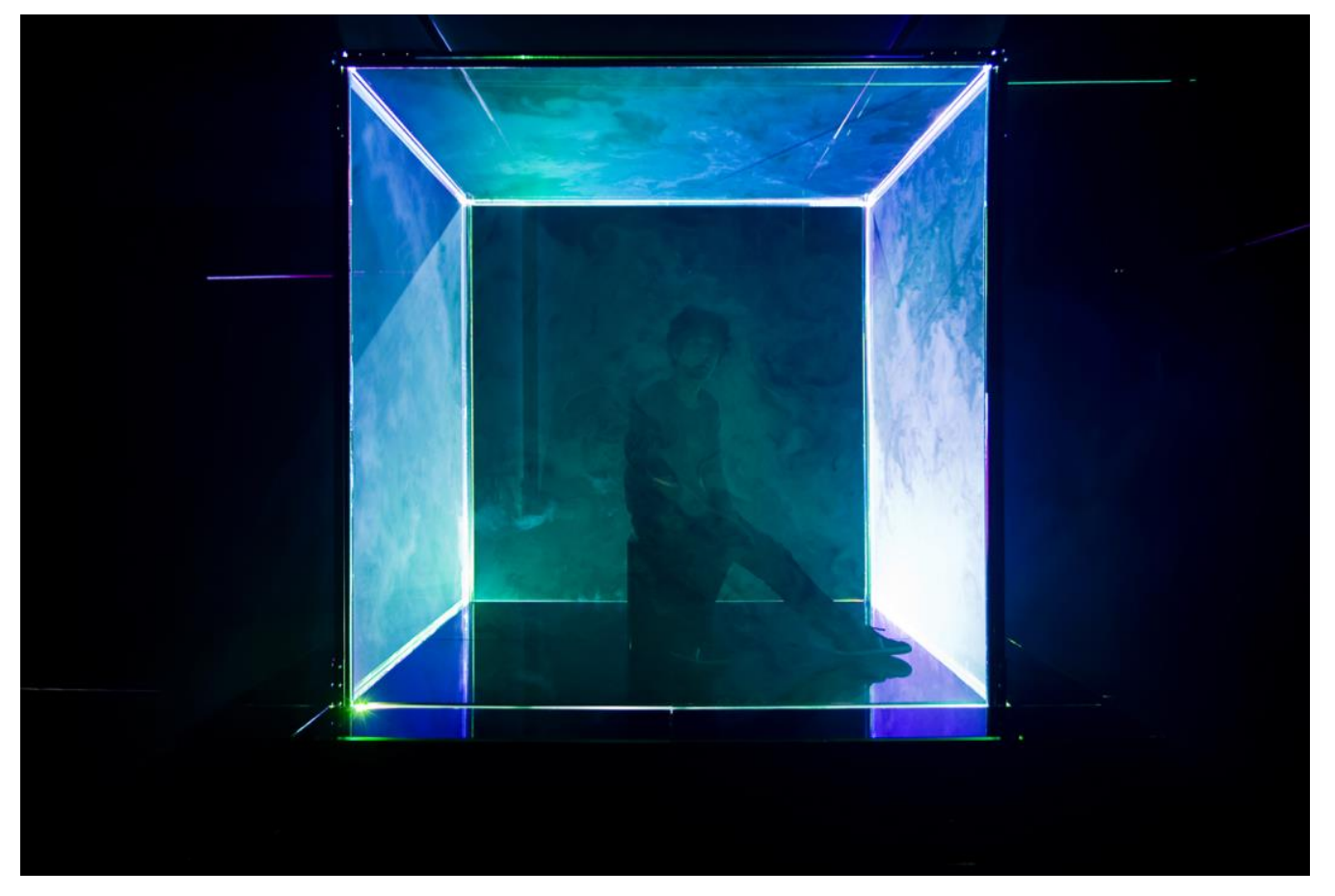

Figure 4. Byungkyu Kim, AT Fleld_Paralyzed Sense, 2012

AT Field_Paralyzed_Sense blocked extraneous light shining into space as much as possible, and light intensity is just bright enough to show light inside space similar to the work by Anthony MicCall mentioned above. AT Field_Paralyzed Sense has technical strengths as the three works analyzed above in light moulding aspect. First, as the AT Field_Paralyzed Sense copfined light in an empty space with cubical frame, it was possible to mould light as a pure substance without a media, the wall, which was used in James Turrell's moulding method forvisualizing light. The second moulding method of AT Field_Paralyzed Sense was able to go beyond the bounds of moulding method of Anthony McCall of leaking light through the opening. Because shapes can be created into three-dimensional with light in a similar manner as the 3D program modeling method when a technology of moulding light into surfaee is used. Lastly, the AT Field_Paralyzed Sense differs from a method of bending light 1nto acurved surface used in the Arcade by Troika as AT Field_Paralyzed Sense used a characteristic of light of shooting in straight line, but such difference in the moulding method can be resolved easily. Because laser can be controlled in any degree into curved line or curved surface with lens that makes the surface modeling possible. Fog was used in space of AT Field_Paralyzed Sense as well to maximize the visibility of light.

\section{Skill Analysis of AT Field_Paralyzed Sense}

The moulding method of light used in AT Field_Paralyzed Sense will be researched minutely in Chapter 3. 


\subsection{Plane of Light_Polygon Mirror Motor}

As the light of laser has the trait of no diffraction and stretching in a straight line, the light has a visual trait of being seen as a line within the space. In addition, the laser also has the trait of being reflected by the reflector such as a mirror. When above two traits of laser are used, the laser can be embodied into two dimensional plane of light. Therefore, when a method to continuously project the line of laser light to the mirror along two dimensional plane is used, the light of laser is shaped into two dimensional plane. In general, a polygon mirror motor is used to shape the light of laser into the plane. The polygon mirror motor is a component of laser scanner or laser printer and it is in shape of regular polygon. In addition, the side projected with the laser is made of mirrors. Therefore, when the laser is projected vertical to the side of polygon mirror fixed vertical to the axis of motor, the light of laser forms two dimensional plane due to the high speed rotation of motor.

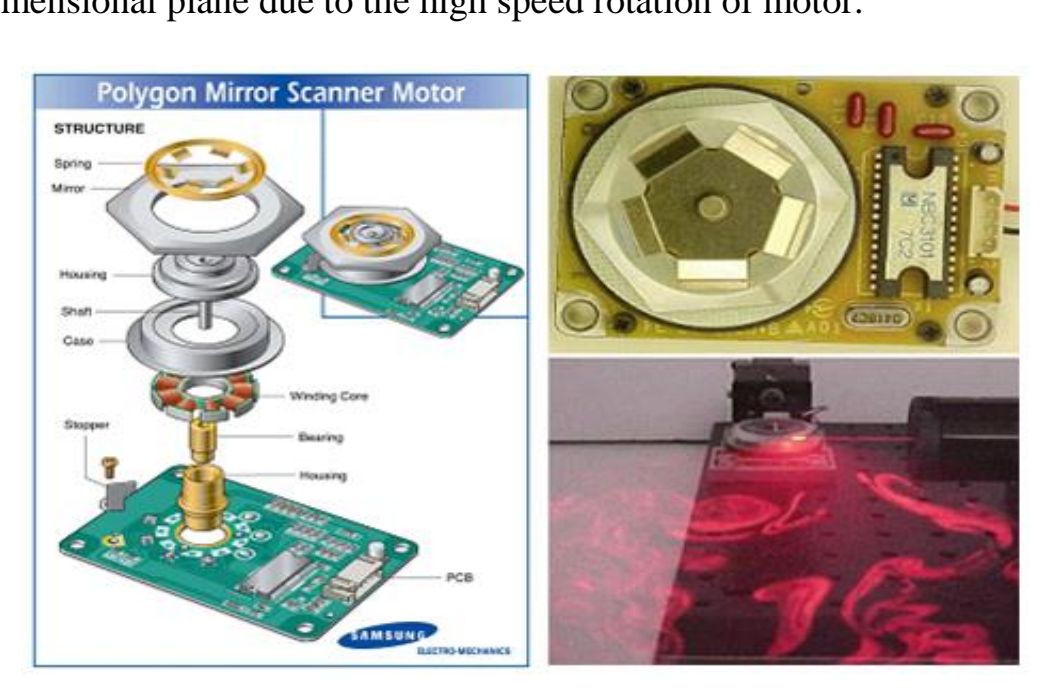

Figure 5. Structure of General Polygon Mirror Motor and Sample of Industrial

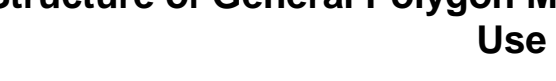

There are two reasons why the polygon mirror motor was custom-manufactured instead of using commercialized ohe. First, the polygon mirror motor purchased in general is produced in small size thus the thickness of reflecting surface which can reflect the light is thin about $5 \mathrm{~mm}$. However, RGB laser used in AT Field_Paralyzed Sense presented the thickness of $5 \mathrm{~mm}, 7 \mathrm{~mm}$ and $12 \mathrm{~mm}$ respectively in its light source thus it was necessary to manufacture a polygon mirror motor with thick reflecting plane. Second, the polygon mirror motor with thick reflecting surface is used in medical devices and sold at about dozens of million won thus it was inadequate considering the limited budget for the production of AT Field_Paralyzed Sense.

The most important manufacturing technology of polygon mirror motor is to adjust all surface angles of mirror attached to each reflecting plane to have the same angle. It is due to the fact that it cannot be visualized as constant and even plane of light if there is a minute distortion in even one reflecting plane. Therefore, as a method to adjust the angle of each plane of manufactured polygon mirror, it shall be manufactured so that the angle can be adjusted by modifying reflecting planes diverted from the axis and horizontal direction of central body. The wire cutting technology was used in order to satisfy above conditions. Each plane separated with the wire cutting is widened and retracted against the axis by two bolts (When the first bolt fixed only to the widened reflecting plane is fastened, the reflecting plane 
is widened as it pushes away the reflecting plane diverted from central body. When the second bolt connected to central body in penetration of the reflecting plane is fastened, the plane retracts toward central body direction).

The projection angle of laser can precisely be controlled as the angle of reflecting plane can be adjusted through above procedure and it enables the creation of constant plane of laser light.

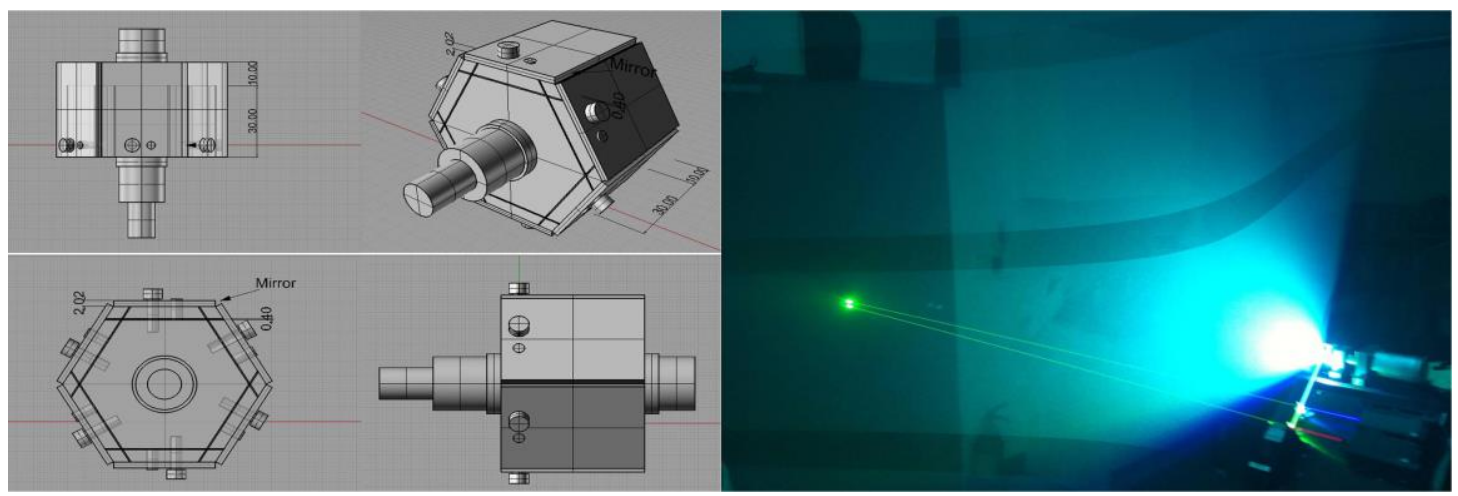

Figure 6. Design Structure of Manufactyred Polygon Mirror and its Test Sight

\subsection{Light Cube}

In order to create a two dimensional plane of light using the polygon mirror motor and embody a three dimensional lignt cube using that plane of light again, a reflecting frame that can trap the laser light must be designed. It is simple to embody a single plane of light. Just attach a mirror to inner plane of frame after manufacturing a rectangular frame. When the light of laser is projected from a cornev of frame after enabling four mirrors to face one another in parallel, the light source of laser is reflected in repetition to plane of four mirrors in sequence. (refer to Figure 7)

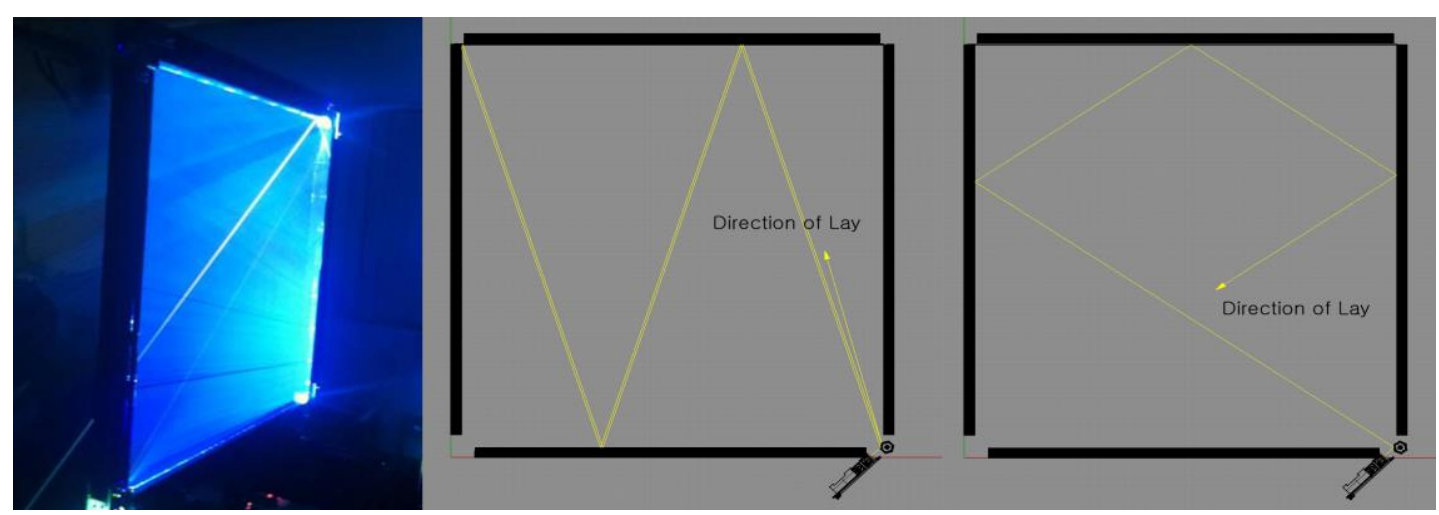

Figure 7. Direction of Laser Light reflected by the Angle of Polygon Mirror and Light Source trapped within Two Dimensional Plane Frame

When four rectangular frames and four lasers are used as illustrated above in order to enable them to face one another, a rectangular shaped laser cube is created excluding the floor and ceiling. Although the floor can be disregarded in a light cube as it is the part where the viewers step on, it is necessary to block the part above the head of viewers with the light. Therefore, when the angle is set to enable four reflecting planes on the top of cube frame to 
reflect the light source of laser in $90^{\circ}$, even the top part can be covered with the plane of laser light. (refer to Figure 8)

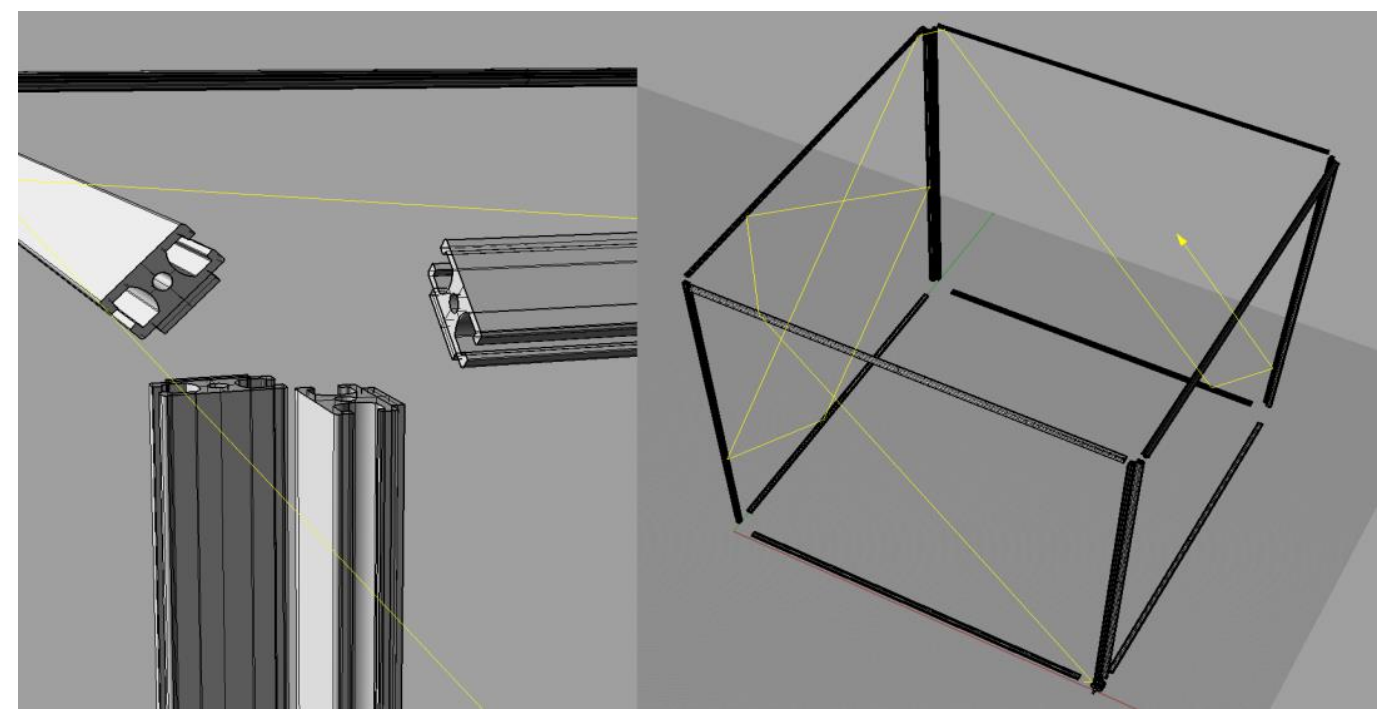

Figure 8. Structure of Three Dimensional reflecting Frame and Straight Direction of Light

\subsection{Hardware Plan}

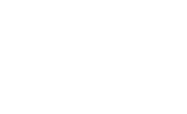

An illustration on the bottom shows a hardware design method of AT Field_Paralyzed Sense. The laser module needs to be placed on the bottom and the frame that confines light by the size of laser module will be placed approximately $45 \mathrm{~cm}$ from the ground. AT Field_Paralyzed Sense was designed into two staircases where viewers can step up in the middle of the structure by hiding the hardware structure.
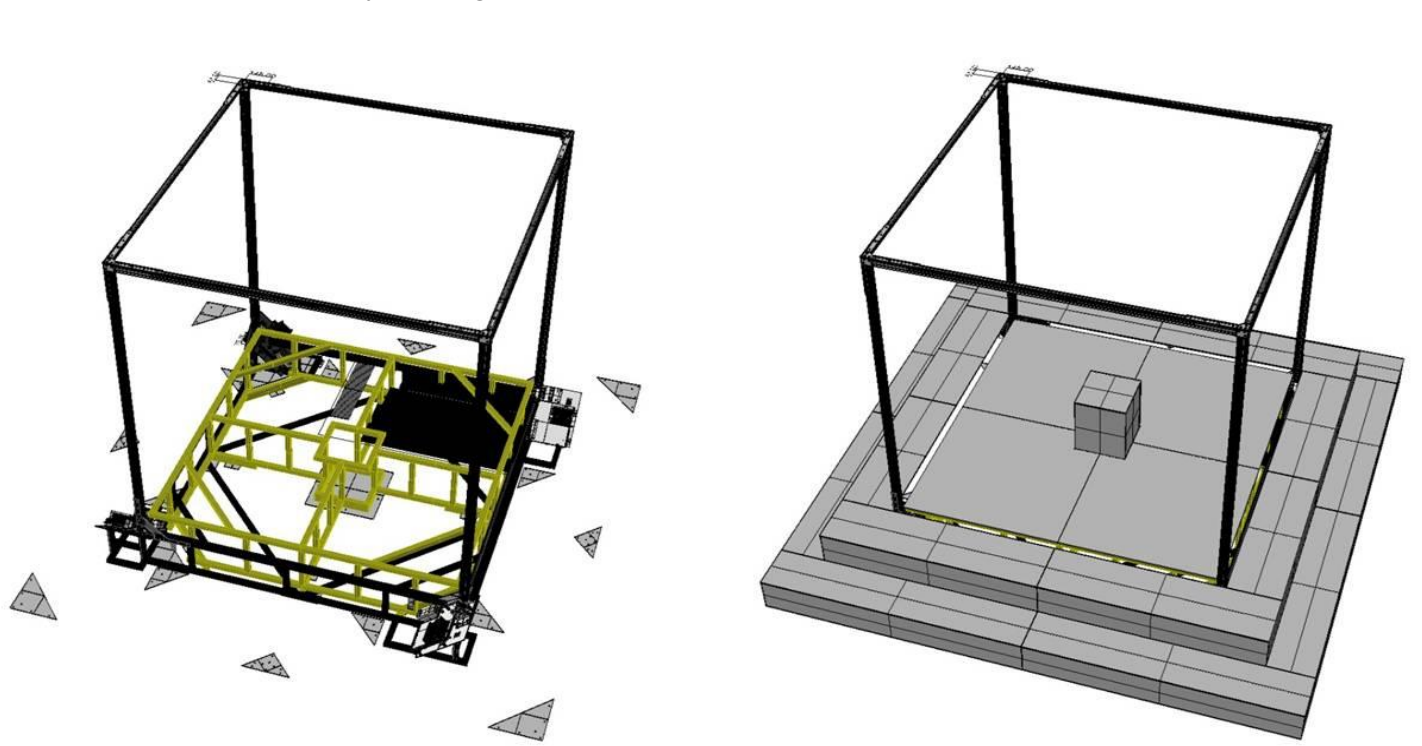

Figure 9. Hardware Plan 


\section{Conclusion}

Unlike objects that are easy to control physically, light is a substance which can only be perceived as a visual form that cannot be touched. Human desire of physically confining light in empty space as an artistic object was visible in a new art genre, called the light art. This study researched works by artists who physically controlled light to create new visual forms and analyzed them. Also, this study analyzed and compared the AT Field_Paralyzed Sense I made with art forms of the same kind.

AT Field_Paralyzed Sense is applied of technique which can visualize and shape the light into the plane. Also, through the plane of light embodiment, it visualized a three dimensional light structure. Although numerous artists have promoted the embodiment work asing the light in the past, it has not reached the quality of physical visualization implemented in AT Field_Paralyzed Sense. Therefore, AT Field-Paralyzed Sense can be considered as an artwork which achieved physical embodiment so substantial to enable fiewers to be under the illusion that the light is a tangible matter. It is suggested that there is a necessity for follow-up studies on new light artworks applying above technique with the use of an attractive substance that is the light.

\section{References}

[1] H. Jung-Hwa, O. Myoung-Ha and K. Hyung-G, "A Study for Figurative Language of Light Art, Korean Society of Basic Design and Art", vol. 11 n6. 2.(2010), pp. 339-348.

[2] S. Jeong-Yeon, "A Study on Design Characteristics of Attistie Space Effected by Light in 'Light and Space Art', Korean Institute of Interior Design, vol. 14 no. 02, (2005), pp. 35-44.

[3] K. Jae-Won, “A Research for Figurative Language of Media Technology Art Using Light- Focusing on Light Art-, Korea Design Knowledge and Industrial Forum, vol. 24, (2009).

[4] C. Paul, "Digital Art", Thamesanld Hudson Atd., (2003), pp. 71-72.

[5] S. v. Wiese, "Kunst LichtKunst; The Artificial Light Spaces by Gruppo T and Zero for Eindhoven", (2006), pp. 448-465.

[6] P. Weibel and G. Jansen, “Editors, Light Art. Artificial Light: Light as A Medium in the Art of the 20th and 21st Centuries", Hatje Cantz, German, (2006), pp. 478-479.

[7] K. Byung-Kyu, "A study on the plane of light embodiment method using the laser", Advanced Science and Technology Letters, vol. 46 (Cames and Graphics 2014), (2014), pp.19-196.

[8] http://terms.naver.com/entfy.non?docId=1155955\&cid=40942\&categoryId=32238

[9] http://en.wikipedia.org/Wiki/Light_art

[10] http://en.wikipedia_org/wiki/Stained_glass

[11] http://jamesturrell.com/artwork/afrum-white/

[12] http://www trøika.uk.com/project/arcades/

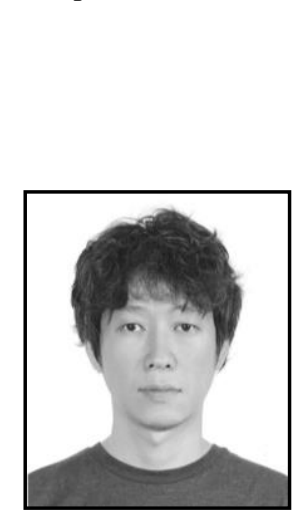

\section{Author}

\section{Kim, Byung-kyu}

Media Artist

Ph.D, Department of Art and Technology, GSAIM, Chung-Ang

University, Media Art 